\title{
Compilation of a Countermeasure Against Instruction-Skip Fault Attacks
}

\author{
Thierno Barry ${ }^{1} \quad$ Damien Couroussé ${ }^{1} \quad$ Bruno Robisson $^{2}$ \\ ${ }^{1}$ CEA - LIST / LIALP \\ Grenoble, France \\ ${ }^{2}$ CEA - Tech / DPACA \\ Gardanne, France \\ firstname.1astname@cea.fr
}

$3^{\text {th }}$ Workshop on Cryptography and Security in Computing Systems

Prague Jan. 20, 2016 


\section{Context}

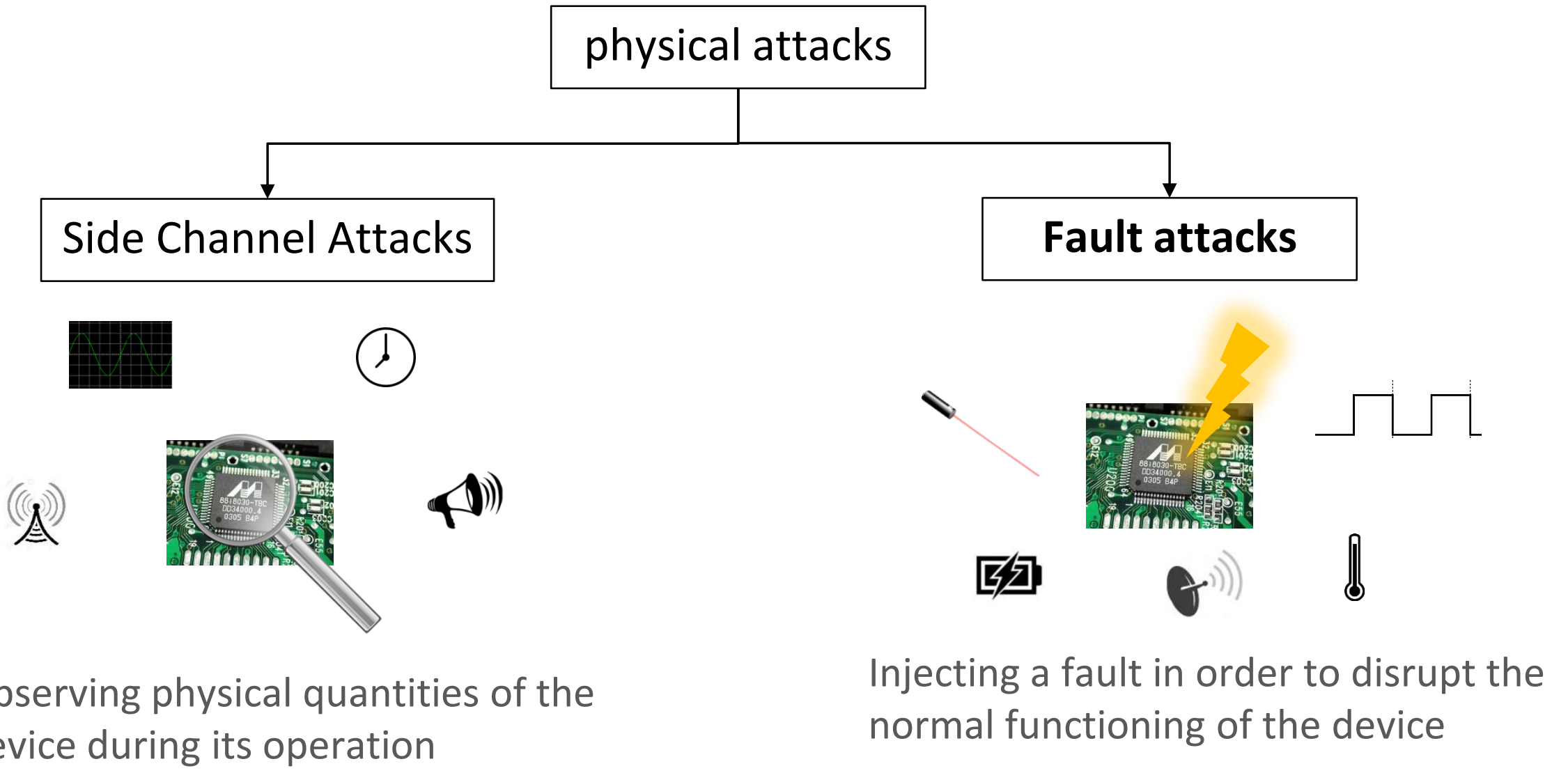

\section{Our objective}

Using compiler techniques to efficiently automate the application of software countermeasures against fault attacks 


\section{Fault Model}

- A fault may occurs at different levels

$\begin{array}{lll}\text { Fault level } & \text { Fault Model } & \text { Countermeasure } \\ - \text { Algorithmic } & >\text { Skip an instruction } & >\text { Instruction redundancy } \\ - \text { Instruction } & >\text { Corrupt a data being transferred } & \\ - \text { Register } & \text { from/to memory } & \\ - \text { Transistor } & \end{array}$

We propose an implementation of the instruction duplication technique

- Based on the scheme proposed and formally verified by [Moro et al. 2014] : "Formal verification of a software countermeasure against instruction skip attacks." 


\section{ceatech}

\section{Instruction Duplication Scheme}

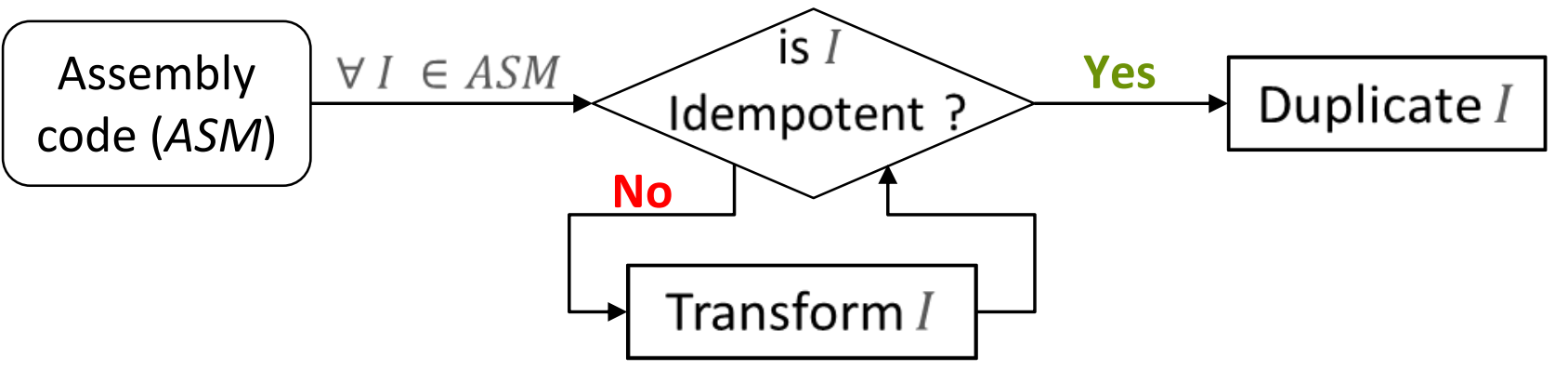

"An instruction is idempotent when it can be re-executed several times with always the same result"

\section{Example:}

is idempotent add R0, R1, R2 $\stackrel{\text { Duplication }}{\longrightarrow} \begin{aligned} & \text { add R0, R1, R2 } \\ & \text { add R0, R1, R2 }\end{aligned}$

Is not idempotent add R1, R1, R2

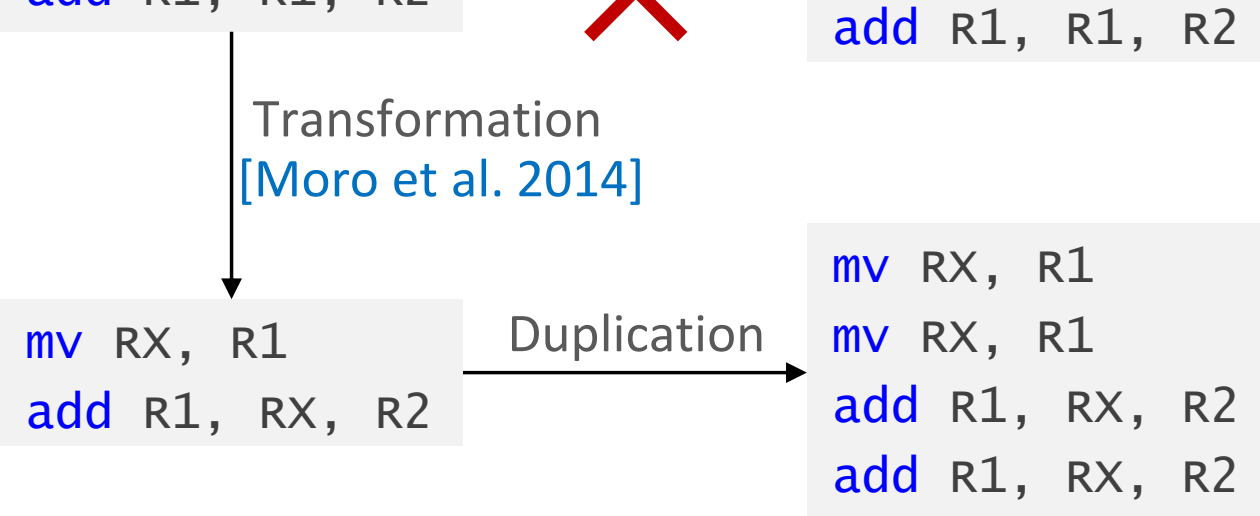

$\longrightarrow$ add R1, R1, R2 add R1, R1, R2

\section{Limitations}

- How to find a free register at the assembly code level ?

- For [Barenghi et al. 2010] the number of free registers are known for their implemented AES

- For [Moro et al. 2014] the use of the ARM scratch register r12

Overhead:

- At least $\times \mathbf{4}$ for each instruction

- [Moro et al. 2014] Reported × 14 for the ARM instruction: um 7 a 1 


\section{ceatech}

\section{Implementation approaches}

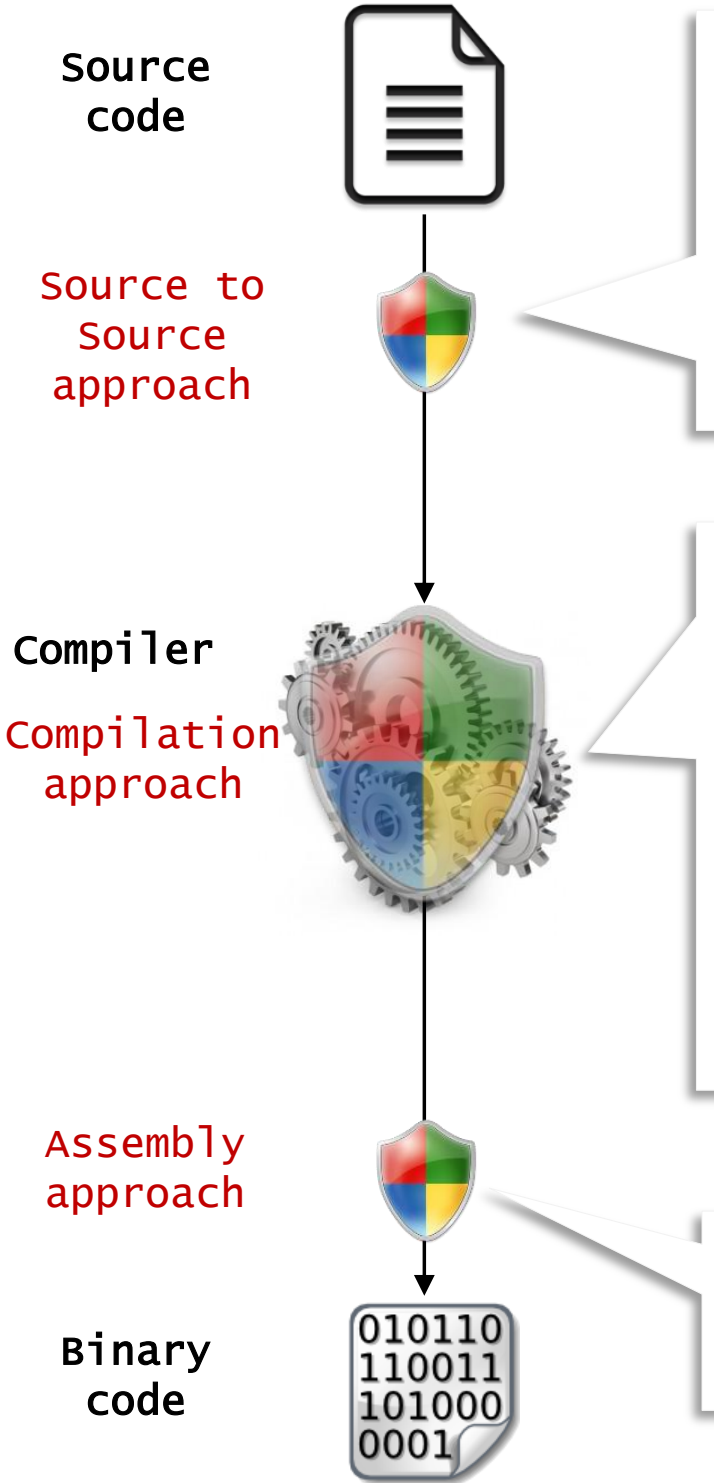

- Security properties cannot be guaranteed after the compilation [Balakrishan et al. 2008]

- Except if the compiler code optimizers are disabled as suggested in [Eldib et al. 2014]

$\rightarrow$ leads to a very high overheads $+400 \%$ in [Lalande et al. 2014]

- Unlike the source to source approach we have control over code optimizers

- Unlike assembly approach we have the benefit of code transformation opportunities provided by the compiler

$\rightarrow$ Allows to reduce the security overhead

- Several transformations need to be performed

$\Rightarrow$ leads to significant overheads [Moro et al. 2014] 


\section{ceatech}

\section{Our approach}

We implemented the instruction duplication inside the LLVM compiler

\begin{tabular}{|c|c|c|c|c|c|c|c|c|c|c|c|}
\hline $\begin{array}{l}\text { Source } \\
\text { Code }\end{array}$ & ס्. & IR & $\frac{n}{d}$ & $\mathbb{R}$ & 들 & 这 & 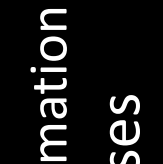 & 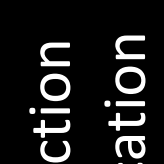 & م. & 임 $\frac{5}{n}$ & $\begin{array}{l}\text { Binary } \\
\text { Code }\end{array}$ \\
\hline & $\frac{1}{\frac{0}{4}}$ & & $\begin{array}{l}\text { O음 } \\
\simeq\end{array}$ & & 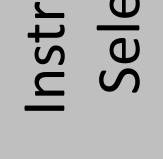 & $\stackrel{\mathscr{\Upsilon}}{\propto} \frac{0}{\bar{\alpha}}$ & 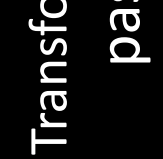 & 들 & 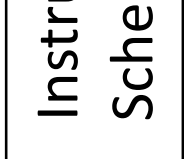 & U & \\
\hline
\end{tabular}




\section{ceatech}

We implemented the instruction duplication inside the LLVM compiler
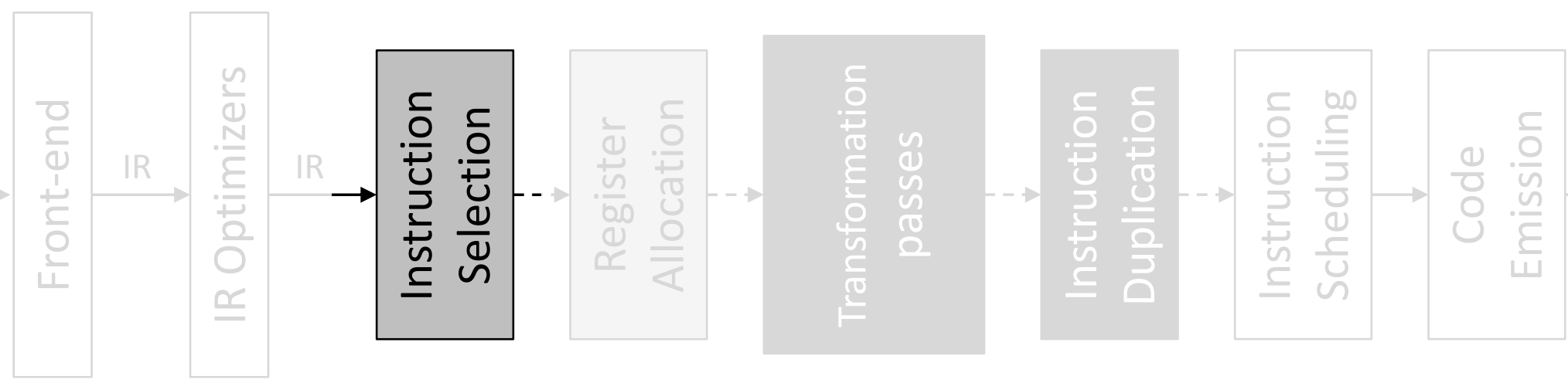

This pass is responsible for selecting the appropriate target instructions for each operation described by the program developer

This pass is modified in such a way that idempotent instructions are the ones privileged during the selection

\section{Example:}

For the operation: $a * b+c$

$\mathrm{mu} \mathbf{l}$ and add are selected instead of $\mathbf{m} \mathbf{l a}$ m7a is not idempotent

But mul and add can be idempotent if the source and destination registers are different 


\section{ceatech}

\section{Our approach}

We implemented the instruction duplication inside the LLVM compiler
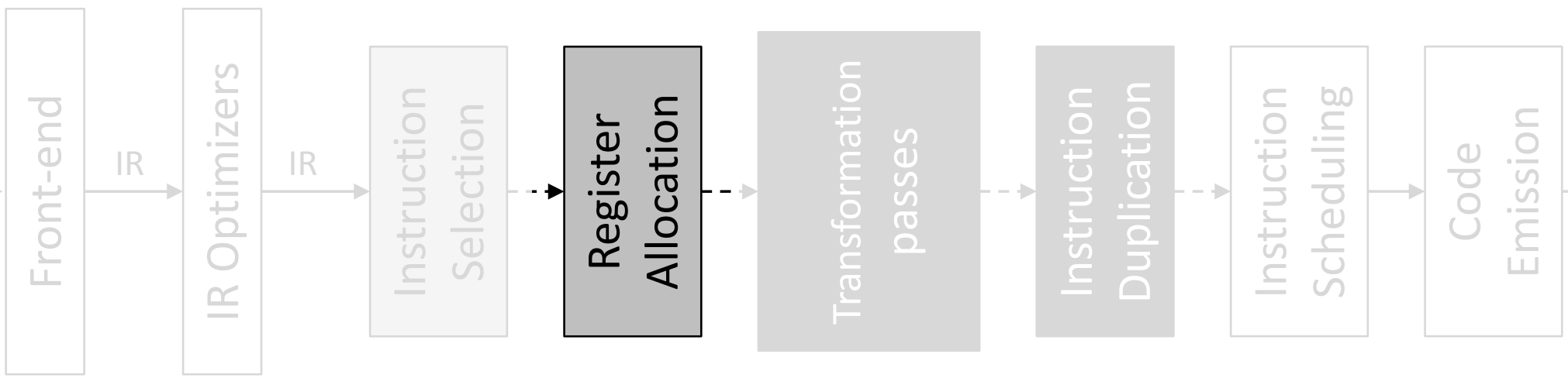

This pass is responsible for mapping the endless number of program variables to a limited number of physical registers

This pass is modified to introduce a constraint so that: destinations registers are always different to sources ones

\section{Example:}

For the operation: $a=b+c$

instead of having: add R0, R0, R1

we have something like: add R0, R1, R2 $\stackrel{\text { Duplication }}{\longrightarrow} \begin{aligned} & \text { add R0, R1, R2 } \\ & \text { add R0, R1, R2 }\end{aligned}$ 
- We implemented the instruction duplication inside the LLVM compiler
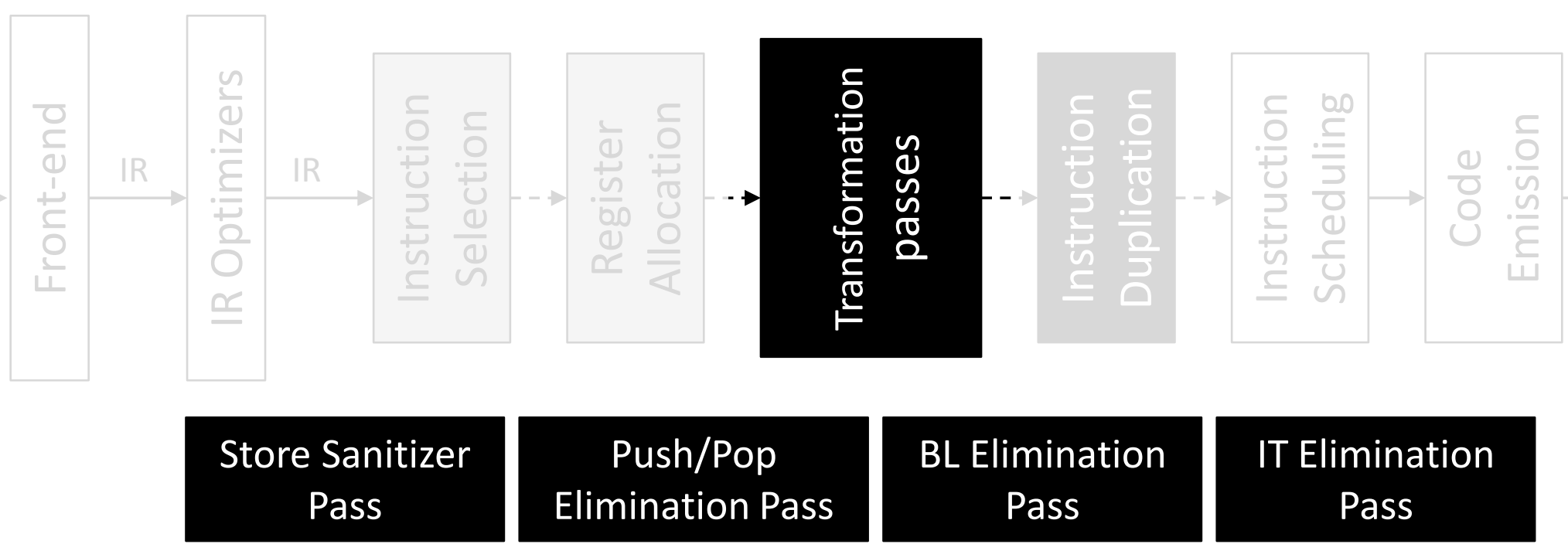
BL Elimination Pass
IT Elimination Pass

The role of these passes is to handle instructions that need special treatments

b1 fun add R0, R1, R2

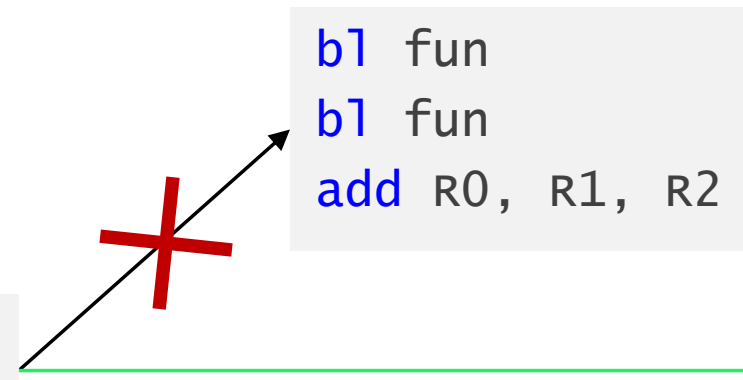

adr RX, retBB add LR, LX, \#1

b fun

b fun

retBB: add $R 0, R 1, R 2$ 


\section{ceatech}

\section{Our approach}

- We implemented the instruction duplication inside the LLVM compiler
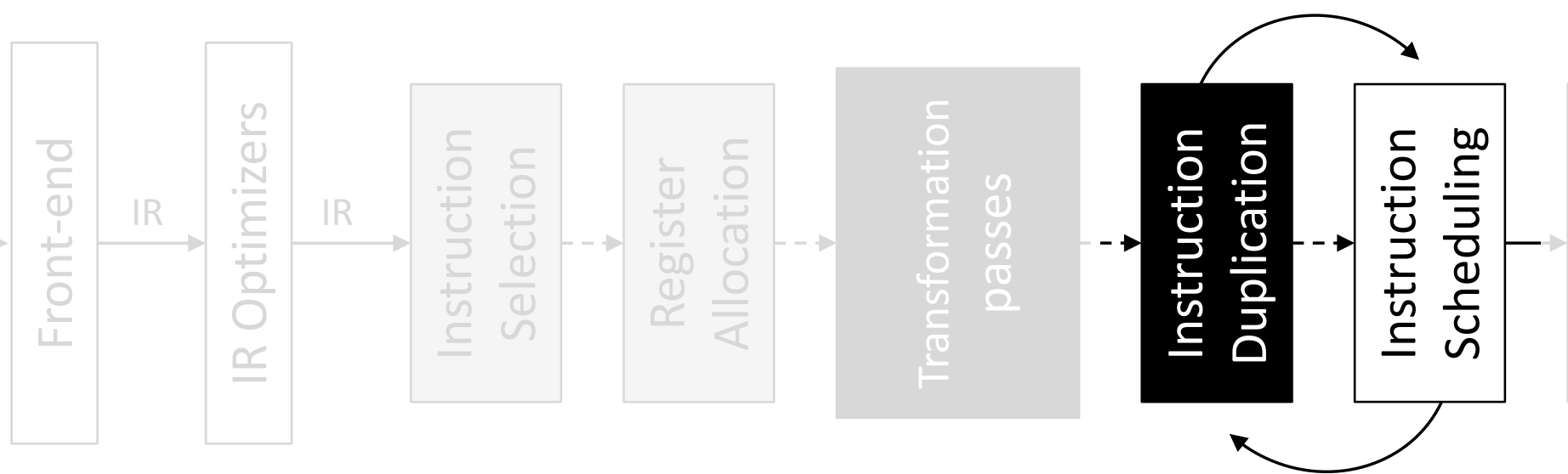

The role of the scheduler is to rearrange the execution order of instruction in order to improve the execution time while preserving the original behavior of the program

\section{Example:}

$$
\begin{aligned}
& \text { add R0, R1, R2 } \\
& \text { add R0, R1, R2 } \\
& 1 d r \text { R3, }[R 1, \# 4] \\
& 1 d r \text { R3, }[R 1, \# 4]
\end{aligned}
$$
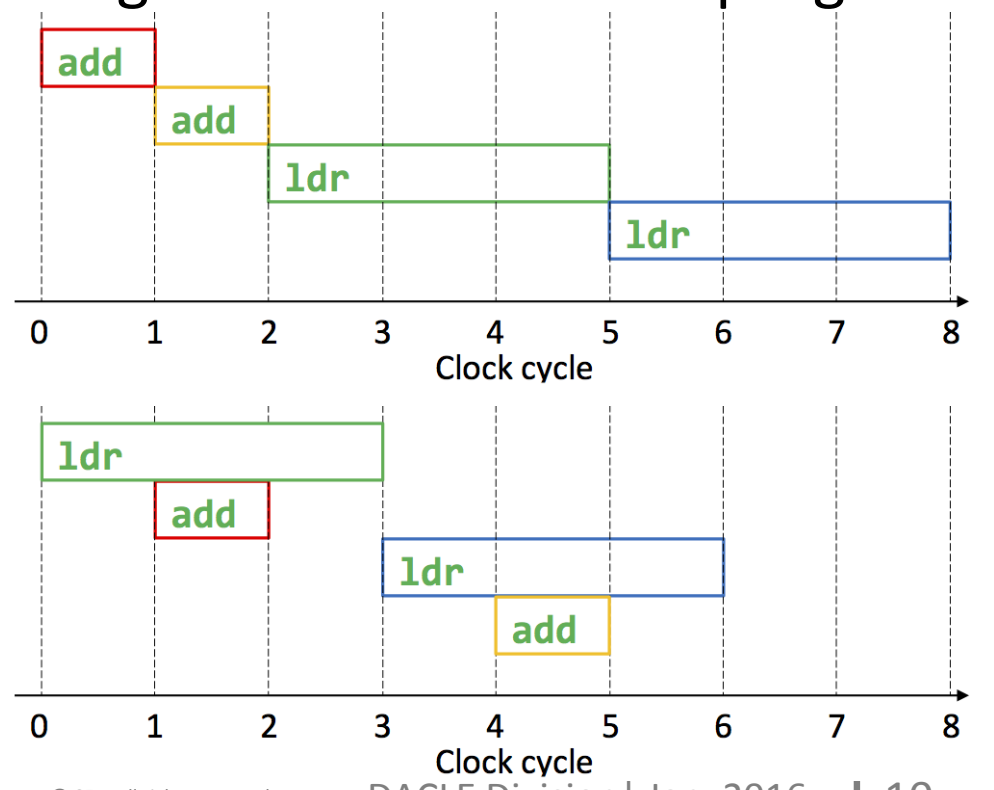


\section{Experimental evaluation}

\begin{tabular}{|l|c|l|l|l|l|l|}
\cline { 2 - 7 } \multicolumn{1}{c|}{} & \multicolumn{2}{l|}{ Unprotected } & \multicolumn{2}{c|}{ Overhead } & \multicolumn{2}{l|}{ Moro et al. 2014} \\
\cline { 2 - 7 } & Cycles & Size & Cycles & Size & Cycles & Size \\
\hline Moro et al.'s AES & 14407 & 11552 & $\times 1.71$ & $\times 1.15$ & $\times 2.14$ & $\times 3.02$ \\
\hline MiBench AES & 1908 & 67644 & $\times 1.76$ & $\times 1.18$ & $\times 2.86$ & $\times 2.90$ \\
\hline
\end{tabular}

Target Architecture: ARM Cortex-M3

- Cycles: clock cycles

- Size: Bytes

- More than $95 \%$ of instructions we generate are idempotent

- Only less than 5\% need to be transformed

1. The impact of the scheduler

Our ARM-based Microcontroller supports both 32-bit and 16-bit instruction set

- The compiler selects 16-bit instructions whenever it is possible 
- We proposed a modified LLVM compiler to efficiently automate the application of the instruction duplication technique

- We illustrated through experimentations the effectiveness of our approach in terms of overheads compared to existing solutions

\title{
Thanks for your attention
}

\section{Compilation of a Countermeasure Against Instruction-Skip Fault Attacks}

\author{
Thierno Barry \\ CEA - LIST / LIALP \\ Grenoble, France \\ thierno.barry@cea.fr \\ http://thiernobarry.fr
}




\section{ceatech}

\section{References}

Balakrishnan, G., \& Reps, T. (2010). Wysinwyx: What you see is not what you execute. ACM Transactions on Programming Languages and Systems (TOPLAS), 32(6), 23.

Eldib, H., Wang, C., Taha, M., \& Schaumont, P. (2014, June). QMS: Evaluating the sidechannel resistance of masked software from source code. In Design Automation Conference (DAC), 2014 51st ACM/EDAC/IEEE (pp. 1-6). IEEE.

Lalande, J. F., Heydemann, K., \& Berthomé, P. (2014). Software countermeasures for control flow integrity of smart card C codes.

In Computer Security-ESORICS 2014 (pp. 200-218). Springer International Publishing.

- Moro, N., Heydemann, K., Encrenaz, E., \& Robisson, B. (2014). Formal verification of a software countermeasure against instruction skip attacks.

Journal of Cryptographic Engineering, 4(3), 145-156.

E Barenghi, A., Breveglieri, L., Koren, I., Pelosi, G., \& Regazzoni, F. (2010, October).

Countermeasures against fault attacks on software implemented AES: effectiveness and cost. In Proceedings of the 5th Workshop on Embedded Systems Security (p. 7). ACM. 